\title{
The impact of imaging technologies on temporomandibular joint disorder diagnosis
}

\author{
Abstracted from \\ Ribeiro-Rotta RF, Marques KD, Pacheco MJ, Leles CR. \\ Do computed tomography and magnetic resonance imaging add to \\ temporomandibular joint disorder treatment? A systematic review of diagnostic efficacy. \\ J Oral Rehabil 2011; 38: 120-135. \\ Address for correspondence: RF Ribeiro-Rotta Department of Oral Medicine, \\ School of Dentistry, Federal University of Goiás, Goiânia, Brazil. E-mail: rejanefrr@gmail.com
}

\section{Question: What is the impact of the information obtained from magnetic resonance imaging (MRI) and computed tomography (CT) images on the temporomandibular joint disorder (TMD) final diagnosis and patient management?}

Data sources Medline, the Cochrane Library databases and reference list of review articles were searched.

Study selection Three examiners selected publications. To be included the publication had to elucidate the diagnostic thinking efficacy and/or therapeutic efficacy of MRI and/or CT in the diagnosis of disc displacement, inflammatory disorders (capsulitis, synovitis) and osteoarthrosis of the temporomandibular joint (TMJ). Publications related to trauma, tumours, systemic diseases, synovial chondromatosis; cadaver studies; narrative literature reviews; and studies of other TMJ imaging methods were excluded.

Data extraction and synthesis Data were extracted by three examiners with disputes being settled by consensus. Study quality was assessed using the QUADAS tool. ${ }^{1}$ No synthesis was performed as only one study was identified.

Results One study was judged relevant. This study evaluated evidence of the efficacy of MRI in the diagnosis of disc position and configuration, disc perforation, joint effusion, and osseous and bone marrow changes in the temporomandibular joint, but no publication reported diagnostic thinking efficacy or therapeutic efficacy.

Conclusions The absence of studies on the therapeutic efficacy of MRI and $C T$ on TMJD reinforces the need for investment in decision-making studies; meanwhile, sectional imaging tests should be prescribed with caution, especially when health budgets are limited.

\section{Commentary}

Diagnosis is central to the practice of dentistry. But how do we diagnose TMD? Diagnosis has traditionally been made by performing clinical examinations and studying radiography records. Clinical diagnosis has improved and diagnostic guidelines are now available. ${ }^{2}$ Imaging diagnosis has also evolved. Traditional planar techniques such as transcranial radiographs have been insufficiently sensitive or specific for TMD diagnosis. But with the development of multiplanar techniques the problem of overprojection of traditional techniques was solved, so that we are now able to see the articular bone structures in detail. In addition, imaging techniques based on nuclear magnetic resonance (MRI) are now available, which permit visualisation of articulations, muscles and most importantly, the articular disc. With these techniques we can thus observe both the structure and function of the TMJ. However, it is important to assess the utility and efficacy of new methods before they become widely adopted or are considered standard. It is no longer sufficient to show that a new diagnostic technology can better depict anatomy or function. Numerous studies have reported findings using these technologies and the aim of this systematic review is to evaluate available evidence with respect to imaging diagnosis and its impact on the therapeutic efficacy for TMD, disc displacement, inflammatory disorders and osteoarthritis.

The authors found a total of 551 studies in a PubMed search and five abstracts in the Cochrane Library database. To these they apply a quality scale for the assessment of studies of diagnostic accuracy (QUADAS ${ }^{3}$ ), excluding all primary research to leave only a systematic review. Thus, the article analysed here is a review of a systematic review. This should be a wake-up call to researchers to improve the quality of reporting of diagnostic test evaluations. Available checklists that may assist them in this task are: STARD ${ }^{4}$ and QUADAS. ${ }^{1,3}$ It might have been valuable to have had the authors report the percentage accomplished for each QUADAS checklist item.

However, attention should be drawn to the fact that even with research into diagnostic imaging methods for TMJ, examination or evaluation guidelines for TMJ still do not exist as they do for clinical evaluations. Further, reproducibility in interpretation of MRI of temporomandibular joints by radiologists is poor. ${ }^{5}$

At a clinical level, the most widely-used parameters for evaluating the utility of a diagnostic technology are diagnostic accuracy and therapeutic impact. ${ }^{6}$ It is important that future studies specify the framework of the research, to better determine the useful scope of the technique. 


\section{TEMPOROMANDIBULAR JOINT DISORDERS}

Does this then mean that we must not use imaging technologies for TMJ diagnosis? No, since it is a fact that tomography, as well as MRI, gives information that complements the clinical examination, but further studies are required to identify whether images exist that are associated with a pathology or dysfunction and whether the value of these technologies lies in identifying or dismissing pathologies, or solely for use as a treatment register.

Sergio Uribe,

School of Dentistry, Universidad Austral de Chile, Valdivia, Chile
1. Whiting P, Rutjes AWS, Reitsma JB, Bossuyt PMM, Kleijnen J. The development of QUADAS: a tool for the quality assessment of diagnostic accuracy included in systematic reviews. BMC Med Res Methodol 2003; 58: 3-25.

2. International RDC-TMD Consortium, Research Diagnostic Criteria for

Temporomandibular Disorders (RDC/TMD). 11/1/2004 International RDC-TMD Consortium. 2011. http://www.rdc-tmdinternational.org/ [Accessed November 2011]

3. 'Bristol University | QUADAS | QUADAS-2.' Bristol University homepage - a place for learning, discovery and enterprise. N.p., 19 June 2011. Web. 10 Nov. 2011. http:// www.bris.ac.uk/quadas/quadas-2/ [Accessed November 2011]

4. Standards for the Reporting of Diagnostic accuracy studies. STARD. N.p., 22 Apr. 2008 Web. 10 Nov. 2011. http://www.stard-statement.org/ [Accessed November 2011]

5. Butzke KW, Batista Chaves KD, Dias da Silveira HE, Dias da Silveira HL. Evaluation of the reproducibility in the interpretation of magnetic resonance images of the temporomandibular joint. Dentomaxillofac Radiol 2010; 39: 157-161.

6. Fryback DG, Thornbury JR. The efficacy of diagnostic imaging. Med Decis Making 1991; 11: 88-94.

Evidence-Based Dentistry (2011) 12, 113-114. doi:10.1038/sj.ebd.6400828 